\title{
Networked Control System - A Survey
}

\author{
M. Brindha \\ Department of ECE, M. V.J College of Engineering, Bangalore-560067, India. \\ E mail:brindha_mo47@yahoo.com \\ Dr J K Mendiratta \\ Department of ECE, M. V.J College of Engineering, Bangalore-560067, India. \\ Email: mendiratta.jk@gmail.com
}

\begin{abstract}
Networked Control System (NCS) is a synthetic application which combines control science, computer science and network technology. It is a kind of feedback control systems wherein the control loops are closed through real time control network. NCS technology is applied in industry control system because of its simple structure, easy maintenance and high reliability. Networked control systems (NCSs) have been gaining popularity with their high potential in widespread applications and becoming realizable with the rapid developments in computer, commun ication and control technologies. This paper reviews the development history of the NCS, and point out the field of further researches.
\end{abstract}

Index Terms - Computer control system, NCS, control Network, network co mmunications, MADB.

\section{INTRODUCTION}

Traditional control systems composed of interconnected controllers, sensors, and actuators have been successfully implemented using a point-to-point architecture. In such architecture system, components are directly wired to various controllers. In modern manufacturing control systems, the goal is to design flexible systems that can accomplish various tasks with small reconfiguration cost. A point-to-point architecture is stagnant from a reconfigurability point of view, since it is easier to build a completely new system than it is to rewire all the system components. Also it does not address the issues, such as interchangeability and reliability that are required of modern control systems, unable to support the industry need for smart sensors and distributed control.

The use of networks as a media to interconnect the different components in an industrial control system is rapidly increasing. For example, in geographically distributed systems, the number and/or location of different sub-systems to control make the use of single wires to interconnect the control system prohibitively expensive. In addition, the flexibility and ease of maintenance of a system using a network to transfer information is appealing. Systems designed in this manner allow for easy modification of the control strategy by re-routing signals, having redundant systems that can be activated automatically, when component failure occurs. And in general, they allow having a highlevel supervis or control over the entire plant.

A major trend in modern industrial and commercial systems is to integrate computing, communication, and control into different levels of machine/factory operations and information processes. Control loops that are closed over a communication network have become more and more common as the hardware devices for networks and network nodes have become cheaper. Over the past decade, major advancements in the area of communication and computer networks have made it possible to include communication in feedback in order to achieve real-time requirements [1]. This gave rise to a new paradigm in control system analysis and design, namely Networked Control System (NCS). Such NCS have received increasing attentions in recent years because of their low cost, easy maintenance and reliability. In today's industry, real-time control systems that need to respond to events under time constraints or deadlines have become increasingly important. At the same time, with the development of network technology, there is a trend in factory, home and automotive equipment towards distributed networking [1]. Un like regular control systems, in networked control systems the synchronization between different sensors, actuators and control units is not guaranteed. Furthermore, there is no guarantee for zero delay or even constant delay in sending information from sensors to the control units and control signals from the control units to the actuators. When there is congestion in the communication network, some packets are dropped to either reduce the queue size in the path or to inform the senders to reduce their transmission rates [5] [6]. In real time systems, particularly control systems, delays or dropped packets may be catastrophic and may cause instability in the control system.

NCS also provides several advantages such as modular and flexible system design, simple and fast implementation, ease of system diagnosis and maintenance. The major advancements in the area of communication and computer networks have made it possible to include them in feedback systems in order to achieve real time requirements. NCS is a special distributed feedback control system where in the control loops are closed through real-time network. It usually includes sensor node, controller node, actuator node, and transmission channel. The typical structure is shown in 
Fig.1. The advantages of NCS are low cost, reliable and ease of installation and maintenance.

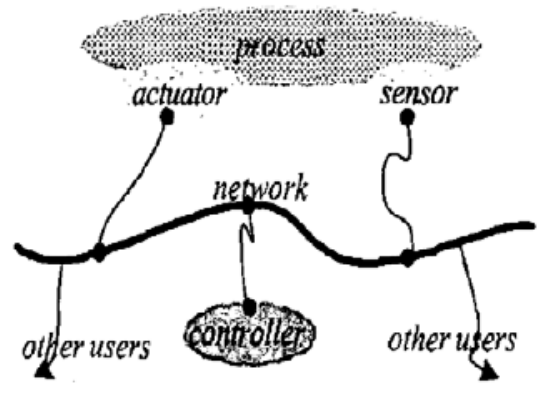

Figure1. Schematic Diagram for an NCS

NCS has brought new challenges in the field of network and control. The main problem is the network latency, multi-channel transmission and packet loss and delays induced by the network. In addition, various network communication protocols are not uniform. So it is necessary to analyze the control science, network parameters, network protocols, scheduling algorithms and integrated approaches to NCS.

In this paper, we discuss NCS arch itecture and how to model NCS based on different parameters involved in the field of communication and control. The next section discusses the issues in the field of controller design and problems associated with communication medium like Network Induced Delay, Data packet drop out, Network scheduling and system stability.

\section{NCS ARCHITECTURE AND MODEL}

NCS is a class of spatially distributed systems wherein the plants, sensors, controllers and actuators are assembled through feedback loops with shared communication network between them. Because of the inherent complexity of such systems, the control issues of NCS have attracted most attention of many researchers with taking into account network-induced delays and/or packet losses, and so on.

NCS model must take into account its special features, such as network delay, non synchronous sampling, packet loss and other multi-packet transmission. The sampling period and the network time delay unify a constant constraint. The network time delay obeys Markov chain, but the analysis of Markov chain transition matrix is cumbersome. Some assumptions made are often not practical. Packet loss is not considered in most of the analysis. Network introduces different forms of time delays between sensors, actuators and controllers. These time delays come from the time sharing of the communication medium as well as the computation time required for physical signal coding and communication processing. The characteristics of time delays could be constant, bounded, or even random [2]. It is well-established in control systems that time delays can degrade a system's performance and even cause system instability. The uncertainty in the magnitude of network and device delays hinders the performance of NCSs and has raised questions concerning what Quality of Control (QoC) can be expected.

\section{ISSUES ON CONTROL STRATEGY}

NCS mostly focuses on how better to deal with the random time-varying delay. It makes control algorithm simple and stable as long as delay and network collisions are not considered. When selecting a communication network for control applications, two key questions related to control performance are: how much time does one message need from the source agent to the destination agent, and how reliable the message transmission is. An in-depth performance evaluation of several popular control networks can be found in [2]. The issues in choosing a controller and problem in scheduling all the systems are analyzed in [3] and a detailed analysis of various issues are discussed in [4]. The control performance of NCS is characterised by sampling rate, controller and network bandwidth. Since a networked agent system is essentially a discrete time system, choosing a proper sampling rate of sensing and actuation data is as important as that in the digital controller design. The sampling rate should depend on the control system bandwidth which is defined as the maximum frequency at which a system will track an input signal in a satisfactory manner. Due to the integral link between the network and control parameters, the selection of the best sampling rate is a compromise.

Communication over shared network causes time delays in various sections of an NCS. These time delays cannot be neglected, especially when the time constant of the controlled plant is short and the order of the plant model is high [5]. They may affect network QOS and degrade control quality of performance. Network delays can degrade the NCS performance and even destabilize the system. From the view of control, delays will degrade system performances of a control system. They can also destabilize the system by reducing the system stability margin [6], [7]. So network delays have caused a lot of attentions. A lot of scholars focus on developing control methodologies to handle this network delay effect on NCS and have made much progress [8].

Assuming that the whole closed-loop NCS consists of a continuous-time plant and a discrete-time controller, in which sensors are time-driven, controller and actuator are event-driven. Then the original model of this NCS is as this:

$$
\begin{aligned}
& x(t)=A x(t)+B u(t) \\
& y(t)=C x(t)
\end{aligned}
$$

Discrete-time controller:

$$
\mathrm{u}(\mathrm{kh})=\mathrm{K} \mathrm{x}(\mathrm{kh})
$$

Where, $x € R^{\prime \prime}, u \in R ', y € R '$, and $A, B, C$ and $K$ are matrices of compatible dimensions. 
An estimator can be used to predict an undelayed plant state and made available for control calculation. Estimators estimates full state of the plant using partial state measurements and also compensate sensor delay. Perturbation control methodology considers the difference between the current plant output values and the most recently transmitted plant output values as a perturbation to the system and limits to this error. The stability of NCS can be proven using Lyapunov approach on the dynamics of the error based on the assumptions like error free communication, fast sampling and noiseless observations. Using Optimal stochastic control, LQG problem is formulated using dynamic programming and optimal controller gain can be achieved. The controller gain can also be updated using fuzzy logic or adaptive control for the best possible performance.

\section{ISSUES ON NETWORKS}

The insertion of a communication network presents some new challenges in NCS modeling, analysis and design. Techniques for modeling the plant/ sensor/ controller/ actuator structure are well known, but techniques for modeling the main characteristics of network communication in an NCS and the interactions between the network and the rest of the systems are still not matured. Therefore the fundamental importance in an analytical and/or a simulation study of NCS is to model and formulate the problem correctly [3], [4]. Because of the presence of network, the ideal assumptions of having (i) equal-d istance sampling, (ii) synchronized control, (iii) non-delayed sensing and (iv) actuation, while modeling the NCS is not true. The existence of networks in control systems brings about the following problems:

\section{A. Network Induced Delay}

In NCS, various delays with variable lengths occur due to sharing of a common network medium, which are called Network Induced Delays. These delays are dependent on configuration of the network and the given system. Delays in the NCS system consist of (a) communication delay between sensors and controllers; $\mathrm{T}_{\mathrm{sc}}$, (b) communication delay between controllers and actuators; $\mathrm{T}_{\mathrm{ca}}$, (c) computation time in the controller; $\mathrm{T}_{\mathrm{c}}$. In practical applications, however, sensor-controller and controller-actuator delays are different and time varying due to network transmission mechanism [9]. Fig. 2 shows the schematic of a feedback control loop with network-induced delays for a single-input and singleoutput (SISO) system. In multi-input and multi-output (MIMO) system, where every sensor and actuator encounters different network induced time delays with espect to the controller sampling instants. Figure 3 shows MIMO system which has ' $M$ ' inputs and ' $R$ ' outputs. Figure 4 shows Network induced delay.

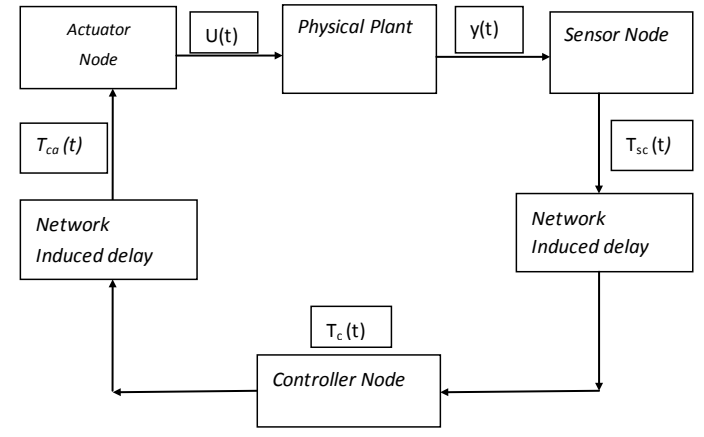

Figure2. Schematic of a feedback control loop with net work-induced delays (SISO)

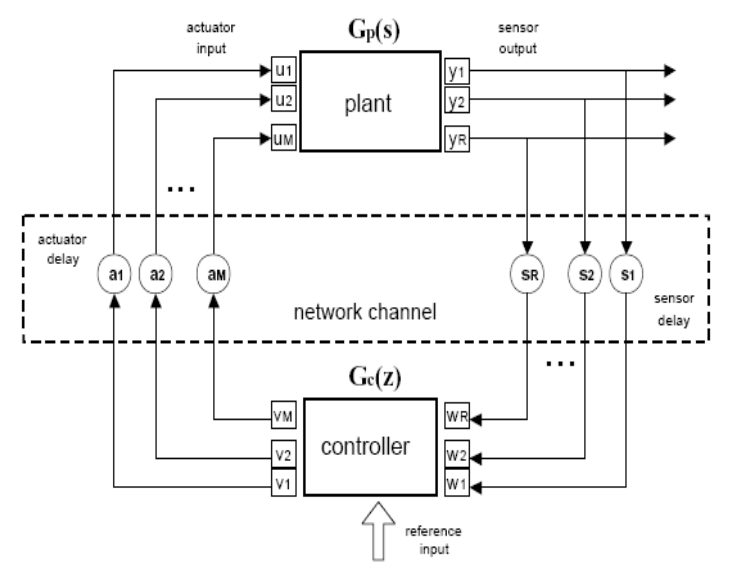

Figure3. Schematic of a feedback control loop with net work-induced delays (MIMO)

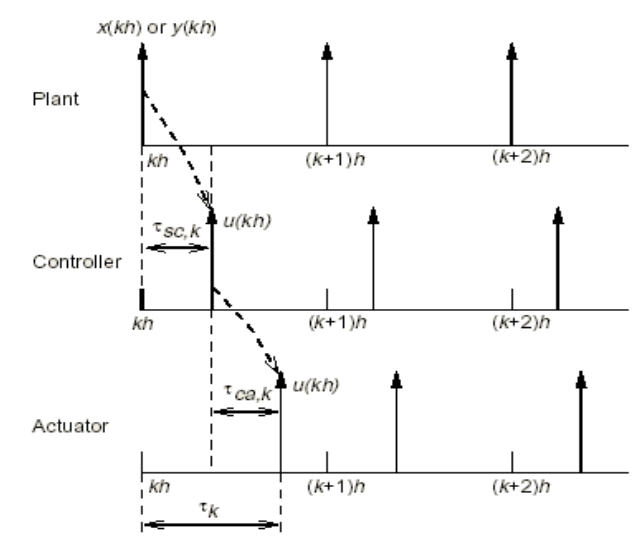

Figure4. Net work Induced Delay

Network Induced delays may vary widely according to the transmission time of messages and the over-head time. The transmission time through the media depends on the network protocols and the over-head time depends on the network scheduling method. NID can degrade the system's performance and even cause system instability [7]. There are two inherent problems in NCS that result from network induced delays. They are Message rejection and Vacant Sampling. 
Message Rejection: When two or more samples from the sensors reach the controller between two sampling instants, then one of the messages is discarded.

Vacant Sampling: When no data arrives to the controller during one sampling period, then the controller uses the previous sample or an interpolated one.

Network delays are usually random because of several sources, for instance, waiting for the network to become idle; a retransmission of urgent data is needed when transmission errors occur; nodes waiting for a random time to avoid a collision at the next try when some networks collisions occur, and so on. As the activities in the system usually are not synchronized with each other, the above listed delays will be random. The transfer delay is independent of the previous delay and has different probability distributions for $T_{s}$, and $T_{c a}$. The system is to be modeled for varying loads and the transitions between different networks load states in the communication network can be modeled with a Markov chain. This delay can degrade the performance of control systems designed without considering it and even destabilize the system. In general, there are two methods to handle the networked-induced delays. One method is to design control algorithms considering the delays, such as in [1-2]; the other is to reduce the delays by feasible scheduling the shared network resource. Recently, part of the research [3-4] on NCSs has focused on how to schedule network resources to make the network-induced delays as small as possible. These research results also have shown that network scheduling plays a, though subordinate but very important, role in NCSs.

\section{B. Sampling Period}

In design of an NCS, finding an appropriate sampling period for each control loop is very crucial. The sampling periods must satisfy two conditions:

1. The stability of the NCS must be guaranteed.

2. The system should be schedulable, that is, all the data transmission must be completed before their deadlines.

The performance of an NCS is highly dependent on the network-sampling period. Increased Network sampling time can improve the performance of the system. However, beyond a critical point, sampling frequency begins to adversely affect NCSs performance, because of network loading, when the number of messages are close to the network saturation limit, message delivery time delays increases and in some cases become unbounded [9]. Optimizing the performance of an NCS can be achieved by balancing the network sampling frequency with the resulting network performance degradation. Due to the interaction of the network and control requirements, the selection of the best sampling period is a compromise. Smaller sampling periods guarantee a better control quality, but result in high frequency communication and may degrade the network quality. The degradation of network quality could further worsen the control quality due to longer time delays when the network traffic is nearly saturated. So selection of sampling period plays an important role in the performance of NCS.

In feedback control systems, the sampled data should be transmitted within a sampling period and stability of the system should be guaranteed in spite of the performance degradation [7]. This certain bound is called a Maximum Allowable Delay Bound (MADB). The Maximum Allowable Delay Bound can be defined as the maximum allowable interval from the instant, when the sensor nodes sense the data fro $m$ a plant to the instant, when actuators output the transferred data to the plant.

For guaranteeing an NCS being stable, the sampling periods must be less than the corresponding maximum allowable delay bounds (MADBs) [4]. The MADBs of the loops can be obtained from stability conditions of the NCS despite of network protocols being used. Now, there have been many studies [13-16] on the stability of NCSs, and some useful results have been obtained. The high sampling rate can increase the network loads, which in turn results in longer delay of the signals. Thus finding a sampling rate that can both tolerate the network induced delay and achieve desired system performance is important in the NCS design.

\section{Data packet Dropout}

The network can be viewed as a web of unreliable data transmission paths. Some packets not only suffer from transmission delay, but also may be lost during transmission for the worst case. Thus, the cause for the packet dropout and the impact on performance of an NCS are required to be considered [10]. Network packet drops occasionally happen in NCSs, when there are node failures or message collisions. Although most network protocols are equipped with transmission retry mechanis ms, they can only re-transmit for a limited time. After this time has expired, packets are dropped. Furthermore, for real time feedback control data such as, sensor measurements and calculated control signals, it may be advantageous to discard the old, un-transmitted message and transmit a new packet, if it becomes available. In this way, controller always receives fresh data for control calculations. Normally, feedback controlled plants can tolerate a certain amount of data loss, but it is valuable to determine whether the system is stable, when only transmitting the packets at a certain rate and to compute acceptable lower bounds on the packet transmission rate.

Network saturation occurs when the network traffic increases beyond a point, when not all messages can be delivered to their destinations in time. Messages may experience long delays due to queuing in buffers, or may be lost due to collisions. For a given sampling frequency, implementing estimation methods in an NCS would reduce network traffic increasing the effective bandwidth of the system.

\section{Network Scheduling}

The performance of NCS depends not only on the 
Control strategies, but also on the scheduling algorithm of network resources. It is more important to investigate the real-time schedule theory of the NCS under conditions, where the network resources are limited. Concepts of network scheduling in NCS are extended from the CPU scheduling in hard real-time computing systems. However, in the cast of network scheduling in NCS, the shared resource becomes the network instead of the CPU processor, and the execution of a real-time task has been replaced by the transmission of a data packet. In addition, network transmissions are normally non-pre-emptive (a transmission, once started is carried out until its completion). Considering that NCS is a digital real-time control system, a controller has to collect message from the sensor, then after processing the message, transfers to the actuator, which directly controls application according to the command. The availability of time on a shared communication network for communication between the sensors, the controller and the actuator is an important factor limiting the performance of an NCS [16]. Therefore, the overall performance of a NCS that consists of several control loops not only depends on the design of control algorithms, but also relies on how to allocate the bandwidth resources, i.e. the scheduling of the shared network resource.

In NCS, various delays with variable lengths occur due to sharing a common network medium. [2]. These delays are dependent on the configurations of the network and the given system. Hence it is necessary to make the delays smaller and bounded, by designing proper scheduling algorithms. The network in an NCS should handle network scheduling algorithms differing in some characteristics from processor scheduling algorithms, such as rate monotonic scheduling algorithms and the deadline monotonic scheduling algorithms. These processor-scheduling algorithms have limitations when applied to NCSs, because a retransmission of periodic data with old values suspended by other urgent data transmissions is meaningless. Network scheduling is an important phenomenon to be considered in the network control system as it may affect the stability of the overall system [11]. By proper design of scheduling algorithms, communication medium can be utilized effectively and efficiently.

The long-time separation between control, communication, and computing communities introduces conservatism and leads to non-optimal solutions. The controller design adopting the variable sampling rate in NCSs has achieved important research results, but most results investigate the stability for a given worst-case interval, which leads to conservative results. This is improved by taking into account a stochastic characterization for the inter-sampling times. In addition, some flexible scheduling schemes dynamically adjust the sampling periods of control tasks during run time, which directly results in sampling jitters within control loops so as to sometimes degrade the QoC. Therefore, the theory and method of controller design must be developed to compensate for sampling jitters.

\section{E. Stability of NCS}

For guaranteeing an NCS being stable, the sampling periods must be less than the corresponding maximum allowable delay bounds (MADBs) [4]. The MADBs of the loops can be obtained from stability conditions of the NCS despite of network protocols being used. The stability of a networked control system should be defined by the performance of both the network and the control system. The Stability is a basic problem in the design of an NCS. The occurrences of transmission events on the network is unknown and often modeled as a random process, e.g., Poisson distribution, and the resulting interval between each access to the network are independent and have an exponential distribution[8] to [11]. The stability of NCS depends not only on the controller but also on the choice of sampling period, scheduling algorithms and various delays occurring in the system.

\section{CONCLUSION AND FUTURE WORK}

Networked control system is related to computer, communication, automatic control and other fields. It has become a research hotspot in recent years. The considerable progress has been made under the joint effort of researchers. However, NCS has not formed a complete theoretical system. A lot of issues have to be studied further. With the embedded technology, NCS nodes will have more and more intelligent, the storage and computing power will become strong. It makes the existing NCS algorithms complex [5]. NCS nodes will have more and more intelligent, the storage and computing power will become strong. The improvement of NCSs performance can be divided into two areas. First, to further guarantee the determinism of transmission time and reduce the end-to-end time delays, device-processing times should be minimized and network protocols can be improved. Second, advanced optimal or robust controller design can overcome the uncertainty in an NCS and achieve the best control performance.

As the nodes are distributed independently, the multi rate sampling is natural for NCSs, but it always brings about some problems: e.g., the constraint of network bandwidth requests better signal quality; redundant signals cause delay, vacant sampling. It is common to adopt the event triggered sampling to deal with the constraint of network bandwidth and the negative impact of redundant signals on the performance of system. A shorter sampling period is preferable to most of the systems, but in some cases, it can be lengthened up to a certain bound, within which, stability of the system is guaranteed [17] -[20]. The high sampling rate can increase the network loads, which in turn results in longer delay of the signals. Thus finding a sampling rate that can both tolerate the network induced delay and achieve desired system performance is important in the NCS design. Networked control system is the future 
distributed system and many studies are going on in the field of network and controller design to improve the performance of NCS.

\section{REFERENCES}

[1] T. C. Yang, "Networked Control System: a brief survey”, Proceedings of IEEE Conference on Control Theory Applications, Vol.153, No.4, July 2006, pp 403 - 412. DOI:10.1049/ip-cta:20050178.

[2] Magdi S. Mahmoud, Abdulla Is mail, "Role of Delays in Networked Control Systems", Proceedings of IEEE Conference on Control System, 2003, pp $40-43$.

[3] Shanbin Li, Zhi Wang, Youxian Sun, "Fundamental Problems of Networked Control System from the view of Control and Scheduling”, Proceedings of IEEE Conference on Control Theory Applications, 2002, pp 2503 - 2508.

[4] Yu jianyong, Yu Shimin, Wang Haiqing, "Survey on the Performance Analysis of Networked Control Systems”, IEEE International Conference on Systems, Man and Cybernetics, 2004. DOI:110.1109/ICSMC.2004.1400997.

[5] Gregory C. Walsh and Hong Ye. "Scheduling of Networked Control Systems", IEEE Control Systems magazine, Volume: 21 Issue: 1, Pages - 57 - 65, Feb. 2001. DOI:10.1109/37.898792.

[6] Kun Ji and Won-jong Kim, “Robust Control for Networked Control Systems with Admissible Parameter Uncertainties" International Journal of Control, Automation, and Systems, vol. 5, no. 4, pp. 372-378, August 2007.

[7] W. Zhang, M.S. Branicky, and S.M. Phillips, "Stability of networked control systems", IEEE Control systems magazine, Vol. 21(1), pages: 84 99, Feb. 2001.E. F. Crawley, J. Malmqvist, S. Östlund and D Brodeur, Rethinking engineering education: The CDIO approach. Springer, 2007. DOI:10.1109/37.898794.

[8] G.C. Walsh, H. Ye, and L. Bushnell, "Stability analysis of networked Control systems", in proceedings of the American Control Conference, Pages: 2876 - 2880, San Diego, June 1999. DOI:10.1109/ICM LC.2002.1174481.

[9] Euler C Martins and Fabio G Jota "Design of Networked control systems with explicit compensation for time delay variations" in IEEE transactions on systems, man and cybernetics, vol 40 no. 3, May 2010.T. Floyd, Digital Fundamentals with PLD Programming, Pearson, 2006. DOI:10.1109/TSMCC. 2009.2036149.

[10] Michael S. Bran icky et al. "Stability of Networked Control Systems: Explicit Analysis of Delay", proceedings of the American Control Conference, Pages: 2352 - 2357, Illinois, June 2000.। Systems”, PhD Thesis, Department of Electrical Engineering and Computer Science, Case Western Reserve University, August 2001. DOI:10.1109/ACC.2000.878601.
[11] Feng- Li Lian, James Moyne, Dawn Tilbury, "Analysis and Modelling of Networked Control Systems: MIMO Case with Multiple Time Delays”, proceedings of the American Control Conference, Arlington, Virginia, June25 - 27, 2001. DOI:10.1109/ACC.2001.945654.

[12] K.J. Astrom and B. Wittenmark, Computer Controlled Systems, Third Edition, Prentice - Hall, 1997.P. Spasov, Microcontroller Technology - The $68 H C 11$ and $68 H C 12,5^{\text {th }}$ ed., Pearson, 2004.

[13] J.Nilsson, "Real-Time Control Systems with Delays." PH.D.Department of Automatic Control, Lund Institute ofTechnology, Lund, Sweden, 1998.R. J. Dirkman and J. Leonard, 68HC11 Microcontroller Laboratory Workbook, Prentice Hall, 1996.

[14] Dong Yue, Qing-Long Han and James Lam, "Networked-based robust Hc, control of systems with uncertainty." Automatica, 41, 2005, pp. 9991007.

[15] W.Zhang. "Stability analysis of networked control systems." PH.D USA: Case Western Reserve University, 2001. DOI:10.1109/87.998034.

[16] Hong Seong and Park, "A scheduling method for network-based control systems." IEEE Transactions on Control Systems Technology, 2002, 10(3), pp.318-330. DOI:10.1109/87.998012.

[17] Walsh, G.C and Hong Ye, "Scheduling ofnetworked control systems." IEEE Control Systems Magazine, 2001, 21(1), pp.57-65. DOI:10.1109/37.898792.

[18] C.L.Liu and J.W.Layland, "Scheduling algorithm for multiprogramming in a hard-real-time environment." Journal of Association for Computing Machinery, 20(1) pp.46-61, January, 1973.

[19] Paul G. Otanez, James R. Moyne, and Dawn M. Tilbury, "Using Dead bands to Reduce Communication in Networked Control Systems", Proceedings of the American Control Conference, Ancharge, May 2002. DOI:10.1109/ACC.2002.1025251.

[20] Feng-Ge Wu, Fu-Chun Sun, De-Qian Xue, Zeng-Qi Sun, "Problems and Strategies of Networked Control Systems", Proceedings of IEEE TENCON'02.DOI:10.1109/TENCON.2002.118265 9. DOI:10.1109/TENCON.2002.1182659.

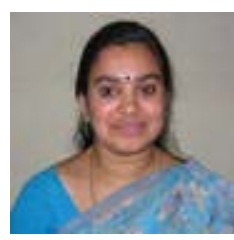

M.Brindha completed Bachelor in Engineering in Electronics and Communication in 2004, Master of Engineering in Electrical Drives and Embedded Control from Anna University, Chennai (2007). She is currently an Associate Professor in the Department of ECE, M.V.J. College of Engineering, Bangalore, India. She has published many journals and attended many Conferences in National and International Level. Her research areas are Embedded Systems, Control system, 
Networking, FPGA Implementation and Algorithms. Email: Brindha_mo47@yahoo.com.

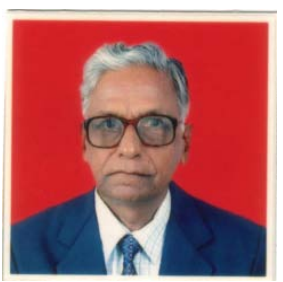

SM (IEEE'93), working as a professor in ECE department in MVJ College of Engineering, has a total publication of 25 papers in national/international journals and conferences. Wide industry and academic experience in teaching and research. Many of the developed products/systems are in manufacture as a regular product of the organizations.Email:mendiratta.jk@gmail.com 